\title{
Implicaciones médicas y nutricionales en insuficiencia cardiaca crónica: fortalezas y limitaciones
}

\author{
Medical and nutritional implications in chronic heart failure: strengths and limitations
}

Lucero Rico-de la Rosa ${ }^{1}$, Miguel Robledo-Valdez', Enrique Cervantes-Pérez ${ }^{1,2 *}$, Gabino Cervantes-Guevara ${ }^{2,3}$, Guillermo A. Cervantes-Cardona ${ }^{4}$, Sol Ramírez-Ochoa ${ }^{5}$, Alejandro González-Ojeda ${ }^{6}$, Clotilde Fuentes-Orozco ${ }^{6}$ y Ma. Fernanda Padilla-Rubio ${ }^{1}$

${ }^{1}$ Departamento de Nutriología Clínica, Instituto Nacional de Ciencias Médicas y Nutrición Salvador Zubirán, Ciudad de México.; ${ }^{2}$ Departamento de Bienestar y Desarrollo Sustentable, Centro Universitario del Norte, Universidad de Guadalajara, Colotlán, Jal.; ${ }^{3}$ Servicio de Gastroenterología, Hospital Civil de Guadalajara Fray Antonio Alcalde, Guadalajara, Jal.; ${ }^{4}$ Departamento de Disciplinas Filosófico, Metodológicas e Instrumentales, Centro Universitario de Ciencias de la Salud, Universidad de Guadalajara, Guadalajara, Jal.; ${ }^{5}$ Servicio de Medicina Interna, Hospital Civil de Guadalajara Fray Antonio Alcalde, Guadalajara, Jal.; ${ }^{6}$ Unidad de Investigación Biomédica 02, UMAE, Hospital de Especialidades, Centro Médico Nacional de Occidente, IMSS, Guadalajara, Jal. México

\begin{abstract}
Resumen
La insuficiencia cardiaca (IC) representa uno de los problemas mundiales de salud pública más importantes, ya que existe un aumento en su prevalencia y se estima que 23 millones de la población mundial viven con este problema. Esta entidad se define por la presencia de anormalidades estructurales y funcionales del músculo cardiaco que conducen a un deterioro en la capacidad del llenado y eyección ventricular. Múltiples comorbilidades se han asociado a un incremento en el riesgo de desarrollo de enfermedades cardiovasculares. La hipertensión se ha reconocido como uno de los factores más importantes, sin embargo, la obesidad, el síndrome metabólico, así como la diabetes, también juegan un papel importante en la aparición de dicha enfermedad. Es frecuente encontrar en pacientes hospitalizados con IC deterioro en el estado nutricional caracterizado principalmente por la presencia de deficiencias nutricionales y sarcopenia, que, en ocasiones, puede progresar y manifestarse como caquexia. Por lo anterior, una evaluación adecuada mediante el uso correcto de herramientas para detección de riesgo nutricional es imperativa, se hace necesaria para prevenir los riesgos que esto implica. Existen múltiples parámetros antropométricos y bioquímicos para definir el estado nutricional de los pacientes hospitalizados, sin embargo, las alteraciones en el volumen sanguíneo presentes en pacientes con IC pueden alterar el resultado de dicha evaluación. Las modificaciones dietéticas en la prevención y tratamiento de diversas enfermedades cardiovasculares mediante un buen apego a patrones de alimentación tales como la dieta DASH (enfoques dietéticos para detener la hipertensión, por sus siglas en inglés) y la dieta mediterránea se han asociado inversamente con la incidencia de IC.
\end{abstract}

Palabras clave: Desnutrición. Resistencia a la insulina. Riesgo nutricional. Insuficiencia cardiaca. Caquexia cardiaca.

\begin{abstract}
Heart failure (HF) is one of the most important global public health problems, as there is an increase in its prevalence and an estimated 23 million of the world's population live with this problem. HF is defined by the presence of structural and func-
\end{abstract}

Correspondencia:

*Enrique Cervantes-Pérez

E-mail: enrique.cervantes@academico.udg.mx
Disponible en internet: 12-04-2021 Arch Cardiol Mex. 2021;91(2):221-228 www.archivoscardiologia.com 1405-9940 / ๑ 2021 Instituto Nacional de Cardiología Ignacio Chávez. Publicado por Permanyer. Este es un artículo open access bajo la licencia CC BY-NC-ND (http://creativecommons.org/licenses/by-nc-nd/4.0/). 
tional abnormalities of the cardiac muscle leading to an impairment of ventricular filling and ejection. Multiple comorbidities have been associated with an increased risk of developing cardiovascular diseases. Hypertension has been recognized as one of the most important factors, however, obesity, metabolic syndrome, as well as diabetes also play an important role in the onset of the disease. It is common to find in decompensated heart failure hospitalized patients an impaired nutritional status characterized mainly by the presence of nutritional deficiencies and sarcopenia, which can sometimes progress to cachexia. Therefore, an adequate evaluation through the correct use of nutritional risk tools should be the cornerstone to the prevention of risks. Multiple anthropometric and biochemical parameters are available to establish the nutritional status of hospitalized patients, however, alterations in blood volume presented in patients with HF may alter the result of such assessment. The effectiveness of dietary modifications in the prevention and treatment of different cardiovascular diseases enhanced by appropriate adherence to eating patterns such as the DASH and Mediterranean diet have been inversely associated with the incidence of HF.

Key words: Malnutrition. Insulin resistance. Nutritional risk. Heart failure. Cardiac cachexia.

\section{Introducción}

La insuficiencia cardiaca (IC) es un trastorno clínico complejo que se caracteriza por disnea, fatiga y retención hídrica, acompañado de signos clínicos relevantes (crepitaciones pulmonares, presión venosa yugular elevada y edema periférico). Además del impacto funcional, la IC está claramente asociada con una mayor probabilidad de hospitalización, así como con una mortalidad elevada'1. Afecta a 6.2 millones de adultos estadounidenses, con una incidencia cercana a 21 por 1,000 habitantes después de los 65 años $^{2}$ y con un costo total de la atención que supera los 30,000 millones de dólares anuales. Las proyecciones estiman que para 2030, más de 8 millones de personas mayores de 18 años se verán afectadas por la enfermedad y que el costo de la atención para entonces será superior a los 70,000 millones de dólares debido al envejecimiento y al crecimiento de la población ${ }^{3}$.

Un análisis del costo de la enfermedad para evaluar el impacto económico de cuatro afecciones cardiacas en México llevado a cabo en 2015 estimó conservadoramente que estas tenían un costo financiero de 96,400 millones de pesos (6,100 millones de dólares). El infarto de miocardio impuso el mayor costo (39,000 millones de pesos/2,500 millones de dólares), seguido de la IC (27,000 millones de pesos/1,700 millones de dólares), hipertensión (22,700 millones de pesos/1,500 millones de dólares) y finalmente, fibrilación auricular (8,400 millones de pesos/532 millones de dólares). La carga de estas cuatro condiciones representó aproximadamente el $4 \%$ del gasto sanitario nacional total ${ }^{4}$.

Por largo tiempo se ha reconocido la asociación que existe entre la presencia de enfermedades crónicas y el deterioro del estado nutricional, así como el impacto negativo que esto genera sobre la calidad de vida en las personas $\mathrm{y}$, por consiguiente, un mayor riesgo de morbilidad y mortalidad 5 .

El estado nutricional y la IC tienen fuertes asociaciones. Las deficiencias de micronutrimentos, como la tiamina y el selenio, por ejemplo, están bien descritas como causa de cardiomiopatía. Sin embargo, la desnutrición es más frecuente en pacientes con IC crónica, a veces progresando a caquexia cardiaca (CC) franca, la cual se caracteriza por desnutrición calórico-proteica con desgaste muscular y edema periférico ${ }^{6}$. La IC crónica es una afección caracterizada por congestión venosa sistémica. La desnutrición en la IC crónica podría estar relacionada con disfunción cardiaca derecha y congestión, que predisponen a edema intestinal, activación inflamatoria y malabsorción, llevando así a desnutrición y a caquexia?.

\section{Insuficiencia cardiaca y deterioro del estado nutricional: enlaces fisiopatológicos}

La IC crónica, el deterioro del estado nutricional y el desgaste muscular comparten muchos procesos fisiopatológicos comunes. Las causas que contribuyen al desgaste en la IC son multifactoriales. La hipoperfusión intestinal y el edema intersticial causan náuseas y anorexia, así como una malabsorción de nutrimentos. La activación neurohormonal con el aumento de la actividad del sistema nervioso simpático y del sistema renina-angiotensina-aldosterona conduce a un aumento del gasto energético basal $y$, por lo tanto, induce un estado catabólico ${ }^{8}$.

El desequilibrio adicional entre el metabolismo anabólico y catabólico en estos pacientes se desencadena por la reducción de los mediadores anabólicos, incluyendo la hormona del crecimiento, el factor de crecimiento tipo insulina 1, la testosterona y la grelina, así 
como el aumento de los mediadores inflamatorios, tales como el factor de necrosis tumoral-alfa (TNF- $\alpha$ ), la interleucina-6 (IL-6) y la proteína $C$ reactiva (PCR), con el consiguiente aumento del estrés oxidativo ${ }^{9}$.

Los pacientes con IC también son más vulnerables a las deficiencias nutricionales debido a la presencia de comorbilidades múltiples como hipertensión, enfermedad arterial coronaria, diabetes mellitus, fibrilación auricular, enfermedad pulmonar crónica, enfermedad renal crónica, enfermedad hepática crónica y anemia ${ }^{10}$. Los pacientes con IC también pueden experimentar empeoramiento del estado nutricional y desgaste al evolucionar a un fenotipo de fragilidad, el cual constituye un síndrome multidimensional, caracterizado por la pérdida de masa corporal magra (sarcopenia), debilidad y disminución de la resistencia al ejercicio físico, que conduce a una disminución de la actividad y pobre respuesta al estrés. La actividad reducida, a su vez, empeora la sarcopenia y la debilidad, que conduce a una tendencia en espiral hacia el deterioro funcional e incrementa el riesgo de muerte ${ }^{11}$. En vista de que no existe una definición estricta de cómo debe evaluarse la fragilidad en la IC, hay una variabilidad significativa en la prevalencia, desde el $21 \%$ en adultos ambulatorios hasta el $70 \%$ en pacientes hospitalizados con IC aguda descompensada $8,12,13$.

\section{Sarcopenia en pacientes con insuficiencia cardiaca}

El término sarcopenia indica una pérdida de la cantidad o calidad del músculo esquelético relacionada con la edad y una disminución de la fuerza muscular y/ o el rendimiento físico no necesariamente asociado con la pérdida de peso $0^{14}$. Von Haehling describe el «desgaste continuo en la IC». Sugiere que, dado que la sarcopenia afecta predominantemente músculos posturales en lugar de músculos no posturales, y la caquexia conduce a una pérdida de tejido graso, así como pérdida de peso, el continuo desgaste en la IC implica que el músculo esquelético se pierde antes que el tejido adiposo $y$, por lo tanto, la sarcopenia precede a la caquexia ${ }^{15}$. Su prevalencia es mayor al $20 \%$ cuando se compara con sujetos sanos de la misma edad. La sarcopenia se caracteriza por anormalidades en el metabolismo energético, junto con disfunción mitocondrial y atrofia de miofibras, con transición del tipo I al tipo $\|^{16}$, y por un proceso progresivo de denervación y reinervación, probablemente secundario a un proceso neuropático crónico que resulta en la pérdida de la unidad motora. Dicho cambio es responsable de una menor capacidad oxidativa y una velocidad de contracción más lenta, lo que causa intolerancia al ejercicio y, a su vez, disminución en la masa del músculo esquelético ${ }^{17}$. Otro factor que contribuye a la sarcopenia en el paciente con IC es la participación de citocinas inflamatorias, las cuales pueden inducir anorexia ${ }^{16}$.

\section{Caquexia cardiaca}

La caquexia asociada a la IC crónica se conoce como CC, cuya prevalencia oscila entre el 5 y el $15 \%$. Se define como la pérdida de al menos un $5 \%$ del peso corporal libre de edema en los 12 meses anteriores (o un índice de masa corporal [IMC] $<20 \mathrm{~kg} / \mathrm{m}^{2}$ ) en pacientes con enfermedad crónica y al menos tres de los siguientes criterios clínicos o de laboratorio: disminución de fuerza muscular, fatiga, anorexia, bajo índice de masa libre de grasa y bioquímica anormal caracterizada por marcadores inflamatorios aumentados (PCR, IL-6), anemia (hemoglobina < $12 \mathrm{~g} / \mathrm{dl}$ ) y/o albúmina sérica baja $(<3.2 \mathrm{~g} / \mathrm{dll})^{18}$. La CC se relaciona con alteraciones hemodinámicas tales como congestión, con la consiguiente proinflamación, malabsorción, anorexia, y la activación neurohormonal. La presencia de CC es un predictor de pronóstico adverso, incluyendo la muerte por todas las causas ${ }^{19}$.

\section{Deficiencias nutricionales en pacientes con insuficiencia cardiaca}

Uno de los mayores riesgos en pacientes con IC es la ingesta calórica disminuida, ya que esta se asocia con una menor calidad de vida y deficiencias nutricionales que podría llevar a eventos cardiovasculares ${ }^{20}$. Las deficiencias crónicas de diversos micronutrimentos pueden favorecer el desarrollo de IC, entre los que se encuentran: coenzima Q10 (CoQ10), tiamina (B1), I-carnitina, taurina y selenio. Estos cinco son componentes esenciales para las vías metabólicas involucradas en la producción de energía, el equilibrio del calcio miocárdico y/o las defensas oxidativas (existen niveles significativamente reducidos en pacientes con IC) ${ }^{21}$.

\section{Coenzima Q10}

El papel más destacado de la CoQ10 es facilitar la producción de trifosfato de adenosina (ATP), participando en reacciones redox dentro de la cadena de transporte de electrones, donde la CoQ10 acepta electrones de los complejos I y II y los transporta al complejo $\mathrm{III}^{22}$. Esta coenzima puede mejorar potencialmente la función cardiaca por medio de una variedad de 
mecanismos, ya que parece ser un potente antioxidante mejorando la disfunción endotelial y posiblemente la producción de ATP cardiaco. Por lo tanto, cuando los niveles de CoQ10 se encuentran bajos, se asocian con mayor severidad de los síntomas de $I^{22}$.

Sander, et al. describen que la CoQ10 mejora la función sistólica en pacientes con IC, existiendo un cambio estadísticamente significativo cuando se compara con placebo ${ }^{23}$. También parece ser un suplemento nutricional seguro y efectivo para el tratamiento de la IC, existiendo una reducción en la morbilidad y mortalidad de aquellos pacientes que ya reciben terapia estándar ${ }^{24}$.

\section{Tiamina (B1)}

La tiamina (B1) es una vitamina hidrosoluble, que juega un papel importante como coenzima en el metabolismo de los carbohidratos. Mediante la adición de magnesio y ATP, la tiamina se convierte en pirofosfato de tiamina por la acción de la tiamina pirofosfocinasa ${ }^{24}$. La deficiencia de esta vitamina puede ocurrir en pacientes con IC debido a una combinación de factores que incluyen desnutrición y aumento en las pérdidas urinarias causadas por la terapia a base de diuréticos (frecuentemente, furosemida) ${ }^{25}$.

Schoenenberger, et al. realizaron un estudio aleatorizado, doble ciego, cruzado, controlado con placebo, que incluyó nueve pacientes con fracción de eyección ventricular izquierda $(\mathrm{FEVI})<40 \%$. Después de 28 días de tratamiento con $300 \mathrm{mg}$ de tiamina vía oral, la FEVI aumentó $32.8 \%$, que fue significativamente $(p=0.024)$ diferente de la FEVI en el grupo placebo (28.8\%). Este estudio mostró que la suplementación con B1 tiene efectos benéficos sobre la función cardiaca en pacientes con IC crónica sintomática ${ }^{26}$.

\section{I-carnitina}

La l-carnitina es un derivado de aminoácidos, sintetizado principalmente a partir de lisina y metionina ${ }^{27}$. Los estudios sugieren que la l-carnitina juega un papel en el uso de ácidos grasos y de glucosa en el miocardio ${ }^{28}$. Una de las manifestaciones causadas por la deficiencia primaria de carnitina en niños es la miocardiopatía dilatada, sin embargo, aún no está claro si se puede observar este tipo de cardiomiopatía en adultos ${ }^{29}$.

Para evaluar el impacto pronóstico de la suplementación con carnitina en la IC, Rizos realizó un ensayo doble ciego, controlado, de 2 g/día de l-carnitina vs. placebo y encontró una disminución de la tasa de mortalidad para los pacientes con I-carnitina que fue estadísticamente significativa a tres años (18\% grupo placebo vs. $3 \%$ grupo l-carnitina, $p<0.04$ ) en pacientes con $I^{30}$.

\section{Taurina}

La deficiencia de taurina a nivel cardiaco genera deterioro en el metabolismo aeróbico y se ha asociado con una reducción en el consumo de oxígeno, elevación en la glucólisis y aumento en la concentración de lactato, así como disminución en la actividad del ATP20. La suplementación de taurina podría proporcionar beneficio antioxidante, ya que este aminoácido es un eliminador del anión hipocloroso y forma taurocloramina (TauCl). La TauCl es un potente antioxidante y agente antiinflamatorio, debido a que inhibe la generación de prostaglandinas, TNF- $\alpha$ e IL-6. La evidencia identifica a la taurina como un agente cardioprotector, ya que puede reducir la aterogénesis y debido a esto juega un papel destacado en el mantenimiento de la fisiología normal de los cardiomiocitos ${ }^{31}$.

\section{Selenio}

El selenio es un micronutrimento esencial para el cuerpo humano, debido a que es necesario para la síntesis de selenoproteínas, las cuales tienen importantes actividades biológicas pleiotrópicas, incluyendo actividad antioxidante, actividad antiinflamatoria y actividad deiodinasa (que es necesaria para la síntesis de la hormona tiroidea activa). La deficiencia de selenio se manifiesta de diversas maneras, tales como epilepsia y lesión cardiovascular que en algunos casos evoluciona a $\mathrm{IC}^{32}$. La enfermedad de Keshan es la presentación clínica clásica de la enfermedad cardiaca secundaria a deficiencia de selenio, que a menudo presenta características clínicas de cardiomiopatía dilatada: choque cardiogénico, arritmias, anormalidades del electrocardiograma, cardiomegalia y/o $\mathrm{IC}^{33}$.

El tratamiento primario de la cardiomiopatía por deficiencia de selenio es la suplementación de dicho micronutriente y la nutrición parenteral. Los pacientes con evidencia demostrada de IC podrían recibir carvedilol, un betabloqueador no selectivo que suprime las especies reactivas de oxígeno con efecto antioxidante y antiinflamatorio ${ }^{21}$.

\section{Evaluación nutricional en pacientes con insuficiencia cardiaca}

Varias herramientas han sido propuestas para evaluar la desnutrición en pacientes con IC crónica y, en 
general, pueden clasificarse como herramientas simples o multidimensionales ${ }^{34}$. Las herramientas simples analizan la desnutrición considerando pruebas de laboratorio y medidas antropométricas (Tabla 1); por otra parte, las herramientas multidimensionales ofrecen una evaluación más completa del estado nutricional mediante la evaluación de una variedad de factores, incluyendo enfermedades agudas, movilidad, comorbilidades y la ingesta alimentaria.

Un estudio reciente en donde se evaluó el estado nutricional de 467 pacientes con IC crónica mostró que la variación en la prevalencia de desnutrición (de cualquier grado y al menos moderada) es mucho mayor entre las herramientas simples (cualquier grado: 6-60\%; al menos moderado: 6-9\%) comparado con herramientas multidimensionales (cualquier grado: $12-29 \%$; al menos moderado: 3-4\%). La puntuación del índice de control nutricional (CONUT), que utiliza albúmina sérica, colesterol total y la cifra de linfocitos plasmáticos, sugirió que muchos más pacientes estaban «desnutridos» en comparación con el índice de riesgo nutricional geriátrico (GNRI), que utiliza albúmina sérica y peso, o el índice nutricional pronóstico (PNI), que combina albúmina sérica con la cuenta total de linfocitos. Hubo un mayor grado de acuerdo en la identificación de los pacientes desnutridos utilizando las herramientas multidimensionales en comparación con las herramientas simples?.

Las diferentes herramientas tienen sus propias fortalezas y debilidades. Entre las herramientas de detección simples, la puntuación CONUT tiene la mayor sensibilidad, pero también tiene la tasa más alta de falsos positivos en la identificación de al menos desnutrición moderada. EI PNI, aunque específico, tiene la mayor tasa de falsos negativos en la identificación de la desnutrición de cualquier grado, por lo que subestima la desnutrición en comparación con otros instrumentos. Esto se debe a que el PNI no tiene una categoría de desnutrición leve y solo identifica a los pacientes con desnutrición al menos moderada. El GNRI parece ser la mejor herramienta de detección simple para la desnutrición en pacientes con IC crónica, pero solo cuando el IMC es $<30^{7}$.

Las herramientas multidimensionales ofrecen una evaluación más completa del estado nutricional y tienen criterios más estrictos para identificar la desnutrición en comparación con las herramientas simples; aunque clasifican una proporción menor de sujetos como desnutridos, es probable que sean más precisos en la detección de la desnutrición. EI MUST (instrumento universal para la investigación de la desnutrición) score y el MNA (minivaloración nutricional) se utilizan comúnmente en
Tabla 1. Parámetros bioquímicos para el diagnóstico de desnutrición

\begin{tabular}{|l|l|}
\hline Albúmina & $\begin{array}{l}\text { Vida media de } 20 \text { días } \\
\text { Niveles bajos en desnutrición. También } \\
\text { en infecciones, quemaduras, sobrecarga } \\
\text { hídrica, insuficiencia hepática, cáncer y } \\
\text { síndrome nefrótico }\end{array}$ \\
\hline Transferrina & $\begin{array}{l}\text { Vida media de } 10 \text { días } \\
\text { Niveles bajos en desnutrición } \\
\text { calórico-proteica, sensible al cambio de } \\
\text { hierro sérico }\end{array}$ \\
\hline Prealbúmina & $\begin{array}{l}\text { Vida media de 2-3 días } \\
\text { Niveles bajos en desnutrición. También } \\
\text { en infecciones e insuficiencia hepática, } \\
\text { y existe un aumento en daño renal }\end{array}$ \\
\hline Proteína C reactiva & $\begin{array}{l}\text { Reactante de fase aguda positivo. Ayuda } \\
\text { a determinar si las proteínas anteriores } \\
\text { se reducen debido a procesos } \\
\text { inflamatorios o a un sustrato } \\
\text { inadecuado, como en la desnutrición }\end{array}$ \\
\hline
\end{tabular}

diferentes entornos: salas de hospital, clínicas, práctica general y hogares de atención ${ }^{35}$.

El MNA-SF, una versión más corta del MNA, es más rápido de completar y tiene una validez y precisión similares a la del MNA en la detección de desnutrición en adultos mayores ${ }^{36}$.

La SGA (Subjective Global Assessment) es la más completa de las tres herramientas multidimensionales. Considera el cambio de peso, los cambios en la dieta, los síntomas gastrointestinales y la capacidad funcional. Además, una proporción significativa de la evaluación depende de los resultados de un examen físico completo. Al igual que el MNA-SF, la SGA también tiene una baja tasa de clasificación errónea en la detección de malnutrición significativa. Sin embargo, la SGA es subjetiva y no es sensible en la detección de la desnutrición en pacientes obesos. También requiere un tiempo significativo para realizar (una media de 20 minutos) ${ }^{7}$.

\section{Intervención nutricional en pacientes con insuficiencia cardiaca}

Los estudios observacionales han demostrado que Ios pacientes con IC tienen habitualmente una ingesta insuficiente de energía y proteínas, y se ha sugerido que las intervenciones nutricionales destinadas a aumentar su ingesta podrían conducir a un mejor mecanismo de adaptación en los desequilibrios anabólicos/ catabólicos causados por el proceso de inflamación y la activación neurohormonal, que son comunes entre los pacientes con $I^{37}$. 


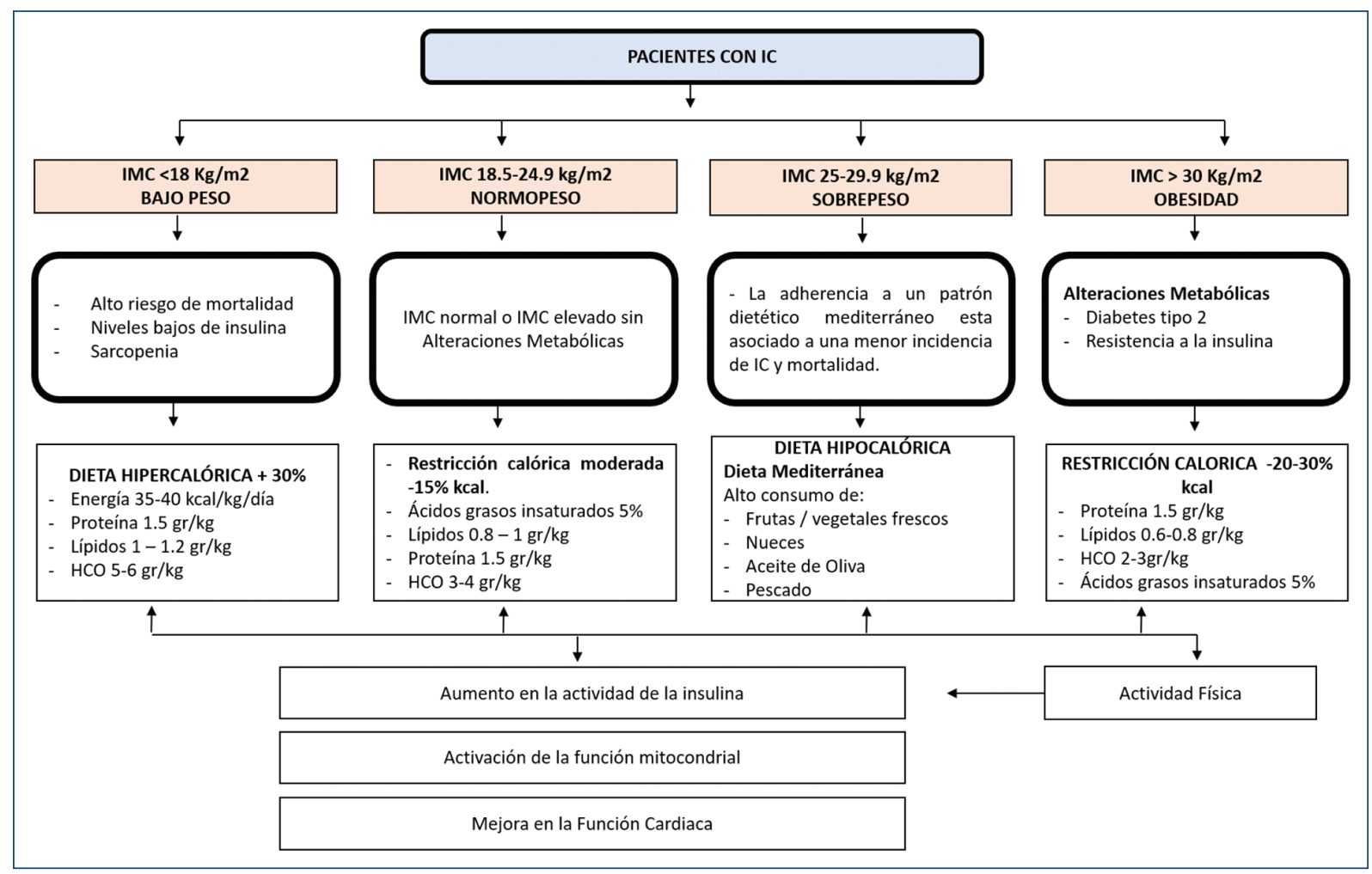

Figura 1. Indicaciones nutricionales en pacientes con insuficiencia cardiaca (IC) según índice de masa corporal (IMC) libre de edema.

$\mathrm{HCO}$ : hidratos de carbono.

La intervención nutricional en estos pacientes debe considerar en primer lugar el IMC (libre de edema) de cualquier individuo, de manera que pueda ser estratificado como bajo, normal y alto, para poder estimar la cantidad de la ingesta calórica, así como la composición de macronutrimentos en la dieta ${ }^{38}$. La figura 1 resume las indicaciones nutricionales en pacientes con IC, según IMC libre de edema.

\section{Recomendaciones dietéticas en pacientes con insuficiencia cardiaca}

La efectividad de las modificaciones dietéticas en la prevención y tratamiento de diversos tipos de enfermedades cardiovasculares está bien reconocida. La dieta DASH (enfoques dietéticos para detener la hipertensión, por sus siglas en inglés), así como la dieta mediterránea, se han asociado de manera inversa con la incidencia de $\mathrm{IC}^{39,40}$.

Ambos patrones de alimentación comparten componentes principales, con la excepción de que la dieta DASH no promueve específicamente el consumo de ácidos grasos monoinsaturados, poliinsaturados y omega 3 , y restringe el consumo de sodio ${ }^{40}$.

Estudios de cohorte multicéntricos evaluaron el efecto de la dieta mediterránea y se encontró una reducción significativa en la evolución de la IC, así como en la preservación de la función ventricular, debido a la mejoría en la presión sistólica y diastólica, al igual que en la función endotelial. Por otro lado, un estudio mostró que el apego adecuado a la dieta DASH tuvo un efecto positivo sobre la función del ventrículo izquierdo y la reducción en la incidencia de $\mathrm{IC}^{38,40}$.

Existe evidencia suficiente que respalda la restricción de sodio en estos pacientes (2-3 g/día), y la restricción de líquidos (1-1.5 l/día) ${ }^{41}$. Diversos estudios con intervenciones a base de dietas bajas en sodio encontraron que las dietas restrictivas $<2 \mathrm{~g}$ de sodio/día aumentan el riesgo de reingreso y mortalidad en pacientes con $\mathrm{IC}^{41}$.

\section{Soporte nutricional}

Los pacientes con IC avanzada suelen presentar desafíos nutricionales, esto se debe al agotamiento de 
reservas que presentan, lo cual genera debilidad y, a menudo, incapacidad para mantener una ingesta oral adecuada, lo que conduce a estados subóptimos de nutrición, que son agravados por la necesidad de ayunar antes de ciertas evaluaciones o procedimientos quirúrgicos. La necesidad de soporte nutricional en pacientes en estado crítico y en aquellos que se someten a cirugía está bien establecida; dichos ensayos se centran en el uso de nutrición enteral (NE) y parenteral (NP). Se ha constatado la seguridad de la NP en pacientes con dispositivos de asistencia ventricular, sin embargo, aún existe controversia acerca del uso temprano vs. tardío. También se ha observado que la ingestión o infusión de una solución de carbohidratos 2-3 horas antes de una cirugía mayor es beneficiosa. Esto previene la resistencia postoperatoria a la insulina asociada con la producción endógena de carbohidratos, disminuye la pérdida muscular postoperatoria y mejora la función inmunitaria ${ }^{42}$.

Por otro lado, cuando el uso de la vía oral es insuficiente o nula, la NE debe intentarse aun cuando se están recibiendo inotrópicos, ya que se ha demostrado que incluso en pequeñas cantidades podría ser benéfica para estimular la mucosa gastrointestinal y de esta manera prevenir la translocación bacteriana ${ }^{42}$.

\section{Conclusiones}

La desnutrición se ha asociado con la aparición de diversas complicaciones en distintas patologías, una de ellas es Ia IC, especialmente en etapas avanzadas, tales como sarcopenia, disfagia, CC, así como la deficiencia de múltiples micronutrimentos que, a largo plazo, pueden evolucionar hacia muchas otras patologías o agravar los síntomas propios de la enfermedad.

El tratamiento nutricional está enfocado en la elaboración de un plan dietético adecuado, así como el tratamiento de comorbilidades presentes en pacientes con IC. La suplementación de multivitamínicos se reserva solo para los pacientes con deficiencias de micronutrimentos comprobadas. El uso de NE y NP está poco estudiado en esta entidad, aun así, cuando la ingesta vía oral está disminuida o es prácticamente nula y el tracto gastrointestinal es funcional, se debe preferir el uso de alimentación enteral sobre la ruta parenteral.

Existe un beneficio potencial de los suplementos nutricionales orales para aumentar el peso corporal en pacientes con IC que están desnutridos 0 en riesgo de desnutrición, así como una intervención dietética individualizada para reducir la mortalidad y la readmisión hospitalaria. Sin embargo, la calidad de la evidencia es baja, y actualmente no se pueden hacer recomendaciones para la práctica clínica. Se necesitan estudios de mejor calidad metodológica y con un mayor número de participantes para determinar la eficacia de las intervenciones nutricionales en pacientes con IC y desnutrición.

\section{Agradecimientos}

Los autores agradecen al Departamento de Nutriología Clínica del Instituto Nacional de Ciencias Médicas y Nutrición Salvador Zubirán.

\section{Financiamiento}

La presente investigación no ha recibido ninguna beca específica de agencias de los sectores público, comercial o sin ánimo de lucro.

\section{Conflictos de intereses}

Los autores declaran no tener conflicto de intereses.

\section{Responsabilidades éticas}

Protección de personas y animales. Los autores declaran que para esta investigación no se han realizado experimentos en seres humanos ni en animales.

Confidencialidad de los datos. Los autores declaran que en este artículo no aparecen datos de pacientes.

Derecho a la privacidad y consentimiento informado. Los autores declaran que en este artículo no aparecen datos de pacientes.

\section{Bibliografía}

1. Normand C, Kaye DM, Povsic TJ, Dickstein K. Beyond pharmacological treatment: an insight into therapies that target specific aspects of heart failure pathophysiology. Lancet. 2019;393:1045-55.

2. Benjamin EJ, Blaha MJ, Chiuve SE, Cushman M, Das SR, Deo R, et al. American Heart Association Statistics Committee and Stroke Statistics Subcommittee. Heart disease and stroke statistics-2017 update: a report from the American Heart Association. Circulation. 2017;135:e146-603.

3. Heidenreich PA, Albert NM, Allen LA, Bluemke DA, Butler J, Fonarow GC, et al. Forecastingthe impact of heart failure in the United States: a policy statement from the American Heart Association. Circ Heart Fail. 2013;6(3):606-19.

4. Stevens B, Pezzullo L, Verdian L, Tomlinson J, Estrada-Aguilar C, George $\mathrm{A}$, et al. The economic burden of hypertension, heart failure, myocardial infarction, and atrial fibrillation in Mexico. Arch Cardiol Mex. 2018;88(3):241-4.

5. Carro A, Panisello JM, Coats AJS. Nutritional status in advanced heart failure and heart transplant patients. Rev Esp Cardiol. 2017;70(8):626-8.

6. Rahman A, Jafry S, Jeejeebhoy K, Nagpal AD, Pisani B, Agarwala R. Malnutrition and cachexia in heart failure. J Parenter Enteral Nutr. 2016;40(4):475-86. 
7. Sze S, Pellicori P, Zhang J, Weston J, Clark AL. Agreement and classification performance of malnutrition tools in patients with chronic hear failure. Curr Dev Nutr. 2020;4(6):nzaa07.

8. Murphy L, Gray A, Joyce E. Anabolism to catabolism: Serologic clues to nutritional status in heart failure. Curr Heart Fail Rep. 2019;16(5):189-200.

9. Raposo André T, Lopes-Santos A. Cardiac cachexia syndrome. Eur Med J Cardiol. 2017:5(1):101-7.

10. Goyal P, Almarzooq ZI, Horn EM, Karas MG, Sobol I, Swaminathan RV et al. Characteristics of hospitalizations for heart failure with preserved ejection fraction. Am J Med. 2016;129(6):635.e15-26.

11. Walker SR, Gill K, Macdonald K, Komenda P, Rigatto C, Sood MM, et al Association of frailty and physical function in patients with non-dialysis CKD: A systematic review. BMC Nephrol. 2013;14:228-37.

12. McNallan SM, Chamberlain AM, Gerber Y, Singh M, Kane RL, Weston SA, et al. Measuring frailty in heart failure: a community perspective. Am Heart J. 2013;166(4):768-74.

13. Vidan MT, Sanchez E, Fernandez-Aviles F, Serra-Rexach JA, Ortiz J, Bueno $\mathrm{H}$. FRAIL-HF, a study to evaluate the clinical complexity of heart failure in nondependent older patients: rationale, methods and baseline characteristics. Clin Cardiol. 2014;37(12):725-32.

14. Cruz-Jentoft AJ, Bahat $G$, Bauer J, Boirie $Y$, Bruyère $O$, Cederholm $T$, et al. Sarcopenia: Revised European consensus on definition and diagnosis. Age Ageing. 2019;48:16-31.

15. Von Haehling S. The wasting continuum in heart failure: From sarcopenia to cachexia. Proc Nutr Soc. 2015;74:367-77.

16. Springer J, Springer JI, Anker SD. Muscle wasting and sarcopenia in heart failure and beyond: update 2017. ESC Hear Fail. 2017;4(4):492-8.

17. Zamboni M, Rossi A, Corzato F, Bambace C, Mazzali G, Fantin F. Sarcopenia, cachexia and congestive heart failure in the elderly. Endocrine, Metab Immune Disord Targets. 2013;13(1):58-67.

18. Evans WJ, Morley JE, Argilés J, Bales C, Baracos V, Guttridge D, et al. Cachexia: A new definition. Clin Nutr. 2008;27(6):793-9.

19. Sato $Y$, Yoshihisa A, Kimishima K, Yokokawa T, Abe S. Prognostic factors in heart failure patients with cardiac cachexia. J Ger Cardiol. 2020:17:26-34.

20. Sciatti E, Lombardi C, Ravera A, Vizzardi E, Bonadei I, Carubelli V, et al. Nutritional deficiency in patients with heart failure. Nutrients. 2016;8(7).

21. Albakri A. Nutritional deficiency cardiomyopathy: A review and pooled analysis of pathophysiology, diagnosis and clinical management. Res Rev Insights. 2019;3(1):1-14.

22. Sharma A, Fonarow GC, Butler J, Ezekowitz JA, Felker GM. Coenzyme Q10 and heart failure. Circ Hear Fail. 2016:9(4):1-9.

23. Sander S, Coleman $\mathrm{CI}$, Patel AA, Kluger J, Michael White C. The impact of coenzyme Q10 on systolic function in patients with chronic heart failure. J Card Fail. 2006;12(6):464-72.

24. Soukoulis V, Dihu JB, Sole M, Anker SD, Cleland J, Fonarow GC, et al. Micronutrient deficiencies. An unmet need in heart failure. J Am Coll Cardiol. 2009;54(18):1660-73.
25. Sica DA. Loop diuretic therapy, thiamine balance, and heart failure. Congest Heart Fail. 2007;13(4):244-7.

26. Schoenenberger AW, Schoenenberger-Berzins R, Auf der Maur C, Suter PM, Vergopoulos A, Erne P. Thiamine supplementation in symptomatic chronic heart failure: study. 2012;101(3):159-64.

27. Siliprandi N, Lisa F Di, Menabò R. Propionyl-L-carnitine: Biochemical significance and possible role in cardiac metabolism. Cardiovasc Drugs Ther. 1991:5(1 Supplement):11-5.

28. Krim SR, Campbell P, Lavie CJ, Ventura H. Micronutrients in chronic heart failure. Curr Heart Fail Rep. 2013;10(1):46-53.

29. Amat di San Filippo C, Taylor MRG, Mestroni L, Botto LD, Longo N. Cardiomyopathy and carnitine deficiency. Mol Genet Metab. 2008;94(2):162-6.

30. Rizos I. Three-year survival of patients with heart failure caused by dilated cardiomyopathy and L-carnitine administration. Am Heart J. 2000;139(2 III):1-4.

31. Zulli A. Taurine in cardiovascular disease. Curr Opin Clin Nutr Metab Care. 2011;14(1):57-60.

32. Amankwah N, Han Z. Cardiomyopathy secondary to selenium deficiency: A review of clinical cases. Open Nutr J. 2018;12(1):74-88.

33. Chen J. An original discovery: Selenium deficiency and Keshan disease (an endemic heart disease). Asia Pac J Clin Nutr. 2012;21(3):320-6.

34. LinH, Zhang H, Lin Z, Li X, Kong X, Sun G. Review of nutritional screening and assessment tools and clinical outcomes in heart failure. Heart Fail Rev. 2016;21:549-65.

35. Guigoz Y. The Mini Nutritional Assessment (MNA) review of the literature-what does it tell us? J Nutr Health Aging. 2006;10:466-85.

36. Kaiser MJ, Bauer JM, Ramsch C, Uter W, Guigoz Y, Cederholm T, et al. Validation of the Mini Nutritional Assessment Short-Form (MNA-SF): a practical tool for identification of nutritional status. J Nutr Health Aging. 2009;13:782-8.

37. Brink M, Anwar A, Delafontaine P. Neurohormonal factors in the development of catabolic/anabolic imbalance and cachexia. Int $\mathrm{J}$ Cardiol. 2002;85(1):111-21.

38. Bianchi VE. Nutrition in chronic heart failure patients: a systematic review. Heart Fail Rev. 2020 Nov;25(6):1017-26.

39. Nguyen HT, Bertoni AG, Nettleton JA, Bluemke DA, Levitan EB, Burke GL. Dash eating pattern is associated with favorable left ventricular function in the multi-ethnic study of atherosclerosis. J Am Coll Nutr. 2012;31(6):401-7.

40. Tektonidis TG, Åkesson A, Gigante B, Wolk A, Larsson SC. Adherence to a Mediterranean diet is associated with reduced risk of heart failure in men. Eur J Heart Fail. 2016;18(3):253-9.

41. Abshire M, Xu J, Baptiste D, Almansa JR, Xu J, Cummings A, et al. Nutritional interventions in heart failure: A systematic review of the literature. J Card Fail. 2015;21(12):989-99.

42. Marelli D. Nutritional support and advanced heart failure. ASAIO J. 2014;60(4):372-3. 